\title{
The Rise and Fall of the Electromagnetic 4-Potential
}

\author{
Eliahu Comay \\ Charactell Ltd., Tel-Aviv, Israel \\ Email: elicomay@post.tau.ac.il
}

How to cite this paper: Comay, E. (2018) The Rise and Fall of the Electromagnetic 4-Potential. Open Access Library Journal, 5: e4979.

https://doi.org/10.4236/oalib.1104979

Received: October 10, 2018

Accepted: November 16, 2018

Published: November 19, 2018

Copyright (c) 2018 by author and Open Access Library Inc.

This work is licensed under the Creative Commons Attribution International License (CC BY 4.0).

http://creativecommons.org/licenses/by/4.0/

\begin{abstract}
The Lienard-Wiechert 4-potential depends on local coordinates and on retarded coordinates of a charge at the source. Therefore, the 4-potential of incoming radiation fields (namely, a photon) cannot be written as a 4 -vector $A_{\mu}(t, \boldsymbol{x})$ which satisfies the locality requirement of fields of a Lagrangian density. This unsolvable problem is the underlying reason for the extremely unusual phenomenon where respectable textbooks make contradictory statements concerning whether the electromagnetic 4-potential $A_{\mu}(t, \boldsymbol{x})$ is a 4 -vector. Moreover, an analysis of well-established experimental data proves that radiation fields and bound fields are inherently different physical objects. These results indicate that the present form of quantum electrodynamics should be revised. It is further proved that in both cases the 4-potential is not a fundamental element of electrodynamics but an auxiliary quantity. For this reason, there are problems with some specific theoretical ideas that pertain to the 4-potential, like gauge transformations, the Dirac monopole theory and the Aharonov-Bohm effects.
\end{abstract}

\section{Subject Areas}

Quantum Mechanics, Theoretical Physics

\section{Keywords}

Special Relativity, Maxwellian Electrodynamics, The Variational Principle, Quantum Theories, 4-Potential

\section{Introduction}

A given physical theory has a mathematical structure that is derived from some expressions and principles. These expressions and principles are regarded as 
postulates that are used for the construction of the entire theory. The goodness of such a theory is determined by a comparison of its predictions with well-established experimental data that belong to the theory's domain of validity.

Once the postulates of a given theory are determined, the rest of the theory is derived from an application of mathematical laws. This general structure of physical theories demonstrates the crucial role of mathematics in the human effort aiming to correctly describe and predict the behavior of physical systems [1]. The present work adheres to this approach. It uses mathematics and experimental data for an examination of the structure of the 4-potential of electromagnetic fields $A_{\mu}(t, \boldsymbol{x})$ and of relativistic 4 -vector requirements.

The variational principle is regarded as a fundamental element of field theories [2] [3] [4] [5]. Another support of this approach states that the variational principle is "the foundation on which virtually all modern theories are predicated" (see [6], p. 353). This principle is adopted here. For the main purpose of this work, let us examine the Lagrangian density of electromagnetic fields (see [2], p. 75 or [7], p. 596)

$$
\mathcal{L}_{E M}=-\frac{1}{16 \pi} F^{\mu v} F_{\mu v}-j^{\mu} A_{\mu}
$$

where $A_{\mu}$ is the fields' 4-potential, $F^{\mu v}$ is the fields tensor which is the 4-curl of the 4-potential (see [2], p. 65 or [7], p. 550)

$$
F_{\mu v}=A_{v, \mu}-A_{\mu, v}
$$

and $j^{\mu}$ is the 4 -current of an electric charge. In this work units where $\hbar=c=1$ are used. Therefore, just one dimension is required and the dimension of length $[L]$ is used. The Minkowski metric is diagonal and its entries are $(1,-1$, $-1,-1)$. Relativistic expressions are written in the standard form. The acronym VE denotes the electrodynamic theory that is constructed on the basis of the variational principle.

One can distinguish between two different classical theories of Maxwellian electrodynamics. The first theory takes the differential equations of Maxwellian fields and the Lorentz law of force as the primary expressions of the theory. The acronym MLE denotes this theory. On the other hand, the well-known textbook [2] proves that MLE can be derived from VE. These theories are not identical. For example, the primary expressions of MLE, namely Maxwell equations and the Lorentz law of force are independent of the 4-potential. Hence, in MLE the 4-potential is an auxiliary mathematical expression whereas in VE it belongs to the theory's fundamental expression (1).

The first term of (1) describes the fields and its second term describes the interaction of the 4-potential with the 4-current of electric charge. This Lagrangian density demonstrates that the 4-potential is a part of the primary expression of VE. The distinction between the two terms of (1) are analogous to that of the philosopher E. Kant who has argued that natural objects have attributes that pertain to "things in themselves" whereas our experience of things has pheno- 
menological features [8]. As stated above, the present work examines properties of the fields $F^{\mu \nu}$ and of their 4-potential $A_{\mu}$. This examination relies on mathematical laws of electrodynamics and on well-known experimental data.

The following simple argument proves the need for the 4-potential in VE. The action has the dimension of $\hbar$. Hence, in the units used herein it is a dimensionless quantity. It follows that all terms of the Lagrangian density (1) must have the dimension $\left[L^{-4}\right]$. In the units used herein charge is dimensionless. Hence, the dimension of charge density is $\left[L^{-3}\right]$. Moreover, charge density is the 0 -component of the 4-current $j^{\mu}$ of (1) (see [2], p. 75). It follows that the interaction term of the Lagrangian density must be a product of charge density and a quantity whose dimension is $\left[L^{-1}\right]$. This is the dimension of the 4-potential. Furthermore, the fact that the Lagrangian density is a Lorentz scalar means that the 4-current $j^{\mu}$ must be contracted with a 4 -vector. These arguments explain why the 4-potential is used. The following article [9] contains a historical review of differences between scientific opinions concerning the role of the 4-potential in electrodynamics.

The second section discusses the significance of the two terms of the electromagnetic Lagrangian density (1) and shows quotations from respectable textbooks that make conflicting statements concerning relativistic properties of the 4-potential. These inconsistencies motivate the analysis presented in this work. The third section proves that bound fields and radiation fields do not represent the same physical entity and points out intrinsic problems with the physical interpretation of the Lienard-Wiechert 4-potential. The fourth section contains a proof of the multi-charge property of radiation fields. The fifth section shows other kinds of problems of the 4-potential. The sixth section discusses results that are derived from the system's Lagrangian density. The seventh section presents problems of theoretical ideas that are connected to the 4-potential: gauge transformation, the Dirac monopole theory and the Aharonov-Bohm (AB) effects. The last section summarized this work.

\section{Problems with the Electromagnetic 4-Potential}

Experimental evidence can be used in an evaluation of the meaning of physical quantities. Let us take the two terms of the electromagnetic Lagrangian density (1), where the first term depends on the fields $F^{\mu \nu}$ and the second term depends on the 4-potential $A_{\mu}$. These terms are used in an examination of a photon emitted from a source at the Andromeda galaxy and measured on planet earth. This photon traveled freely for about one million years. It means that during this time interval the second term of (1) was irrelevant to the photon's motion.

Another issue is the calculation of the energy-momentum tensor of electromagnetic fields (see [2], pp. 86-89 or [7], pp. 601-608). This calculation is based on the first term of (1) and ignores the second term which depends on the electromagnetic 4-potential. 
These arguments indicate that the electromagnetic fields $F^{\mu v}$ play a more significant role in electrodynamics, because there are cases where the fields are needed whereas the 4-potential can be ignored. Therefore, problematic issues of the 4-potential may have a secondary effect on the consistency of the theoretical structure of electrodynamics.

Let us see what some respectable textbooks say on relativistic attributes of the electromagnetic 4-potential $A_{\mu}(t, \boldsymbol{x})$ and derive self-evident conclusions from this information. The main issue is to see if textbooks make equivalent statements on the following problem: is the 4-potential $A_{\mu}(t, \boldsymbol{x})$ of electromagnetic fields a genuine 4-vector? Evidently, a definition of a 4 -vector is needed for this end. A well-known textbook (see [2], p. 15) says about this issue:

" $A$ set of four quantities $A^{0}, A^{1}, A^{2}, A^{3}$ which transform like the components of the radius four-vector $x^{\mu}$ under transformations of the four-dimensional coordinate system is called a four-dimensional vector (four-vector) $A^{\mu}$."

This statement is called below the 4-vector definition. It is used in the examination of the 4-potential $A_{\mu}(t, \boldsymbol{x})$.

The following quotations from respectable textbooks illustrate the problem with the electromagnetic 4-potential that are related to the 4-vector definition. (Equation numbers are the same as those of the quoted textbooks.)

1) One textbook says: Obviously, Lorentz covariance requires that the potentials $\Phi$ and $\boldsymbol{A}$ form a 4-vector potential

$$
A^{\alpha}=(\Phi, \boldsymbol{A})^{\prime \prime}
$$

(see [7], p. 549). Later, this textbook treats the 4-potential (11.132) as a genuine 4 -vector.

2) Another textbook says: Thus the action function of a charge in an electromagnetic field has the form

$$
S=\int_{a}^{b}\left(-m c \mathrm{~d} s-\frac{e}{c} A_{\mu} \mathrm{d} x^{\mu}\right) .
$$

The three space components of the four-vector $A^{\alpha}$ form a three-dimensional vector $\boldsymbol{A}$ called the vector potential of the field. The time component is called the scalar potential; we denote it by $A^{0}=\Phi$. Thus

$$
A^{\alpha}=(\Phi, \boldsymbol{A})^{\prime \prime}
$$

(see [2], p. 48).

3) A third textbook makes the following statement: "In short,

$$
A_{\mu}=(\Phi, \boldsymbol{A})
$$

is a four-vector. What we call the scalar and vector potentials are really different aspects of the same physical thing. They belong together. And if they are kept together the relativistic invariance of the world is obvious. We call $A_{\mu}$ the four-potential' (see [10], p. 25-8).

4) By contrast, a fourth textbook examines the 4-potential of a photon and states that "the fact that $A^{0}$ vanishes in all Lorentz frames shows vividly that 
$A^{\mu}$ cannot be a four-vector" (see [3], p. 251).

5) Similarly, another textbook analyzes the 4-potential of electromagnetic fields and states that "we lose manifest Lorentz and gauge covariance" (see [4], p. 73).

As a matter of fact, the textbooks [3] [4] show how particular gauge transformations of the 4-potential can be used for amending the problem. However, the above mentioned 4-vector definition proves that these transformations only show how one can proceed in spite of the fact that the 4-potential $A_{\mu}(t, \boldsymbol{x})$ is not a 4-vector.

The foregoing quotations clearly demonstrate that well-known textbooks make inconsistent statements concerning the problem of whether the electromagnetic 4-potential $A_{\mu}(t, \boldsymbol{x})$ is a genuine 4-vector. This state of affairs stimulates an adequate analysis of this issue. The rest of this work is dedicated to this objective. The arguments presented below adhere to the two fundamental elements of theoretical physics: they abide by mathematical laws and rely on relevant experimental data.

\section{Radiation Fields and Bound Fields}

The following analysis examines the data of the hydrogen atom and proves that radiation fields and bound fields are different physical entities. It is well known that quantum mechanics properly describes atomic systems. Therefore, this theory is used below as a reliable description of the data (see [11], pp. 60-90).

Let us take the $1 \mathrm{~s}$ ground state of the hydrogen atom. Properties of this state are time-independent, and they are derived from an analysis of the electron's quantum state. The stability of the ground state means that it is an eigenfunction of the Hamiltonian. The spatial angular momentum and the parity of the $1 \mathrm{~s}$ ground state are $0^{+}$.

Now let us consider the interaction of the hydrogen atom ground state with an incoming photon whose energy equals the difference between the energy of the $2 p$ state and the $1 \mathrm{~s}$ state of this atom. This interaction induces a transition from the 1 s ground state to the $2 \mathrm{p}$ state (see [11], p. 264). The spatial angular momentum and the parity of the $2 \mathrm{p}$ state are $1^{-}$. It follows that the spin and the parity of the photon are $j^{\pi}=1^{-}$(see also the photon data here [12]).

A calculation of the spin and parity of each quantum state of the hydrogen atom is based on the electronic state of the atom whereas electromagnetic bound fields make no contribution to this calculation (see [11], pp. 60-90). Hence, if bound fields represent a quantum particle then the spin and the parity of such a particle are $0^{+}$. This is inconsistent with the photon data. Now, radiation fields are associated with a photon, which is a well-known quantum particle. These conflicting spin and parity values prove that whether or not bound fields represent a genuine quantum particle, radiation fields and bound fields are inherently different physical entities (see also [13]).

The same results are obtained from an examination of the system's energy. Indeed, let us slightly modify the opening statement of the previous paragraph. 
A calculation of the energy of each quantum state of the hydrogen atom is based on the electronic state of the atom whereas electromagnetic bound fields make no contribution to this calculation (see [11], pp. 60-90). Hence, the energy of bound fields vanishes. It means that the spin, parity and energy of bound fields are the same as the corresponding quantities of the vacuum.

This result shows another difference between MLE and VE. Indeed, in MLE the energy-momentum tensor represents the fields energy (see [2], pp. 86-89 or [7], pp. 601-608). It indicates that in this theory the fields energy does not vanish whereas in $\mathrm{VE}$, atomic bound fields have no energy.

Let us find out how this conclusion affects the meaning of the electromagnetic 4-potential. The Lienard-Wiechert 4-potential $A_{\mu}$ of a pointlike charge $e$ is (see [2], p. 174; [7], p. 656)

$$
A_{\mu}=e \frac{v_{\mu}}{R^{\alpha} v_{\alpha}} .
$$

Here $R^{\mu}$ denotes the 4 -vector from the retarded position of the charge to the measurement point, and $v^{\mu}$ denotes the charge's retarded velocity. This 4-potential yields bound fields as well as radiation fields (see [2], p. 175; [7], p. 657). The following argument proves that these attributes of the 4-potential (3) mean that it cannot represent a genuine quantum particle.

Indeed, it should be pointed out that Wigner's analysis of the unitary representations of the inhomogeneous Lorentz group proves that a massive quantum particle has a well-defined mass and spin whereas a massless quantum particle has two components of helicity [14] [15]. This evidence means that the expression of the Lienard-Wiechert 4-potential (3) cannot be consistently related to a physical object, because the energy and the spin values of bound fields and of radiation fields that are derived from this $A_{\mu}$ take contradictory values.

\section{Multi-Charge Properties of Radiation Fields}

Consider the two radiating systems of Figure 1. Figure 1(a) shows a single charge $q$ that moves uniformly along a circle which is embedded in the $(x, y)$ plane and its center coincides with the origin of coordinates. The point $p$ lies on the $z$-axis at the radiation zone. The circular motion of the charge $q$ proves that

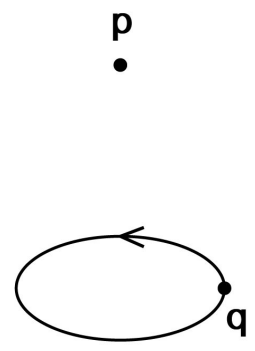

(a)

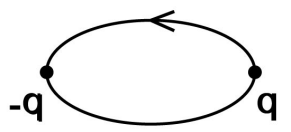

(b)

Figure 1. Two radiating systems. (a) A charge $q$ moves uniformly along a circle; (b) Two charges, $\pm q$ move uniformly along a circle. See text. 
it accelerates towards the center of the circle. This acceleration $\boldsymbol{a}$ indicates that radiation fields do not vanish at point $p$ (see [2], p. 175; [7], p. 657).

Let us examine the radiation fields $E, B$ at point $p$ and compare the fields of Figure 1(a) with those of Figure 1(b). In Figure 1(b) there are two charges $\pm q$, which are located at two antipodal points of the circle. These charges move along the circle with the same velocity as that of the charge $q$ of Figure 1(a). For point $p$, the retarded time of the charge $+q$ is the same as that of the charge $-q$. Well known formulas of radiation fields prove that, at point $p$, the electric field and the magnetic field of the charge $+q$ are the same as those of the charge $-q$ (see [2], p. 175; [7], p. 657). It means that at point $p$, the radiation fields $\boldsymbol{E}, \boldsymbol{B}$ of Figure 1 (b) are twice as strong as those of Figure $1(\mathrm{~A})$. Now, the Poynting vector (see [2], p. 81 or [7], p. 237)

$$
\boldsymbol{S}=\boldsymbol{E} \times \boldsymbol{B} / 4 \pi
$$

describes the energy current of electromagnetic fields. This vector proves that point $p$, the energy current of Figure 1(b) is four times greater than that of Figure 1(a). Furthermore, since the same frequency holds for the fields of the two cases, one concludes that at the vicinity of point $p$, the number of photons of Figure 1(b) is four times greater than the number of photons of Figure 1(a). It means that the actual radiation emitted by a system of charges is not the sum of radiation emitted by individual charges. The foregoing example proves the following important conclusion:

The radiation emitted from a system of charges is a multi-charge effect.

It is shown below that this conclusion is relevant to the problem of the electromagnetic 4-potential $A_{\mu}(t, \boldsymbol{x})$.

\section{Further Inconsistencies of the 4-Potential}

Section 3 proves the existence of problematic issues of the 4-potentials. A general rule says that if a theory contains one inconsistent element then it is likely that other inconsistencies can be found in that theory. The following lines prove that the 4-potential belongs to this case.

Let us examine the electromagnetic Lagrangian density (1). The general structure of a Lagrangian density of a field function $\Psi$ is

$$
\mathcal{L}\left(\Psi(x), \partial \Psi(x) / \partial x^{\mu}\right),
$$

where $x$ denotes the four space-time coordinates $x \equiv(t, \boldsymbol{x})$. This form indicates local properties of the functions on which the Lagrangian density depends. As stated in the following textbook, this is a general attribute of presently accepted field theories: "all field theories used in current theories of elementary particles have Lagrangians of this form" (see [3], p. 300). Hereafter this form is called the locality of the Lagrangian density. The generalization of (5) to the case of several kinds of independent fields is straightforward (see [4], p. 15). Let us compare this form of the Lagrangian density with mathematical properties of the electromagnetic fields and of their 4-potential. 
The electromagnetic fields satisfy Maxwell equations which are partial differential equations with respect to the four space-time coordinates $(t, \boldsymbol{x})$. A unique solution is obtained for any appropriate initial/boundary value problem [7]. Therefore, these fields satisfy the locality of the Lagrangian density. On the other hand, the Lienard-Wiechert 4-potential (3) is a 4-vector which depends on the local four space-time coordinates $(t, x)$ and on the charge's retarded coordinates as well. Hence, it is inconsistent with the locality of the Lagrangian density. In particular, radiation fields carry energy and the principle of energy conservation shows that these fields are derived from a 4-potential that depends on accelerating charges at the radiating source.

The Lienard-Wiechert formula for the 4-potential (3) proves that in the case of radiation fields, the retarded coordinates may be very far away from the relevant spatial region at the vicinity of $(t, \boldsymbol{x})$. For example, consider a laboratory that contains an electron of a hydrogen atom. This electron interacts with a photon that has been radiated from a source at the Andromeda galaxy. In this case, the distance between the hydrogen's electron and the photon's source is of the order of $10^{32}$ times the relevant size of the hydrogen atom. This is yet another indication showing that the Lienard-Wiechert 4-potential (3) is inconsistent with the locality of the Lagrangian density (5).

It is shown above that the Lienard-Wiechert 4-potential (3) is inconsistent with the local form $A_{\mu}(t, \boldsymbol{x})$ which is required for the electromagnetic Lagrangian density (5). Furthermore, section 4 proves that radiation is a multi-charge effect. Hence, the actual number of independent variables of the photon's 4-potential is much larger than the 4 coordinates $(t, x)$ used in (5). It follows that the problem is uncorrectable and a 4-vector of the form $A_{\mu}(t, x)$ cannot be used in a reliable mathematical expression for the 4-potential of an incoming radiation.

\section{Discussion}

The analysis presented in Section 3 proves that radiation fields and bound fields do not represent the same physical entity. Therefore, these fields should be treated separately. One element of this issue applies to the important case of the Lagrangian density of a system of an electrically charged Dirac particle and electromagnetic fields (see [4], p. 84, [5], p. 78).

$$
\mathcal{L}=\bar{\psi}\left[\gamma^{\mu}\left(i \partial_{\mu}-e A_{\mu}\right)-m\right] \psi-\frac{1}{16 \pi} F^{\mu v} F_{\mu v} .
$$

This is a quantum expression which is an extension of (1). It contains additional terms that represent the charged matter. Here the interaction of a charged particle with an incoming radiation and the interaction of a charged particle with the fields of other charged particles of the same system should be treated separately. The following discussion briefly shows some aspects of this issue.

Let us examine the interaction of the system's charges with an incoming radiation. It is proved in sections 3 and 5 that the 4-potential of radiation fields 
$A_{\mu}(t, \boldsymbol{x})$ cannot be used in a physically acceptable Lagrangian density. It follows that contrary to a fundamental requirement, the interaction term of the Lagrangian density (1)

$$
\mathcal{L}_{\text {Int }}=-j^{\mu} A_{\mu}
$$

is unacceptable. The same is true with the interaction term of (6). This problem can, however, be settled if the fields term of the Lagrangians (1) and (6)

$$
\mathcal{L}_{\text {Fields }}=-\frac{1}{16 \pi} F^{\mu v} F_{\mu v}
$$

is regarded as the radiation's primary expression and the 4-potential of the interaction term is treated as an auxiliary quantity. As pointed out in Section 5, electromagnetic fields satisfy the locality of the Lagrangian density. In this case the right form of the 4-potential can be reconstructed in every frame on the basis of the Lorentz transformation of the fields [16]. It is interesting to point out that this procedure has the important advantage of directly yielding a 4-potential whose form is required by quantum electrodynamics (QED), namely

$$
A_{0}=0, \nabla \cdot \boldsymbol{A}=0
$$

(see [4], p. 73). It means that the correct result is obtained without resorting to gauge transformation (see below subsection 7.1).

Let us turn to bound fields. It is proved in Section 3 that the spin and the parity of these fields are $j^{\pi}=0^{+}$and that they do not contribute to the system's energy. These attributes are the same as those of the vacuum. It follows that bound fields cannot represent a physical particle altogether. And indeed, the Darwin Lagrangian shows that bound fields of a system of charges can be replaced by a mechanical-like expression where the coordinates and the velocities of each pair of charges take their instantaneous value and $\boldsymbol{n}_{i j}$ denotes a unit vector from the position of the ith particle to that of the jth particle

$$
L_{\text {Darwin, int }}=-\sum_{j} \sum_{i>j} \frac{e_{j} e_{i}}{R_{i j}}+\sum_{j} \sum_{i>j} \frac{e_{j} e_{i}}{2 R_{i j}}\left[\boldsymbol{v}_{j} \cdot \boldsymbol{v}_{i}+\left(\boldsymbol{v}_{j} \cdot \boldsymbol{n}_{i j}\right)\left(\boldsymbol{v}_{i} \cdot \boldsymbol{n}_{i j}\right)\right]
$$

(see [2], pp. 179-182, [7], pp. 593-595). This expression is independent of the electromagnetic fields and of their 4-potential. In the quantum case of Dirac particles, this expression boils down to the Breit interaction (see [17], pp. 170, 195). Here the velocity operator $\boldsymbol{\alpha}$ of a Dirac particle (see [18], p. 11) replaces the particle's velocity $v$. The equal time attribute of the Breit interaction is consistent with the standard quantum mechanical practice of atomic state calculations [17]. Indeed, atomic state calculations determine the Hamiltonian's eigenfunctions. These eigenfunctions can be written in the form of the time-independent Heisenberg picture (see [4], pp. 5-8).

The mechanical-like Lagrangian of Darwin and Breit proves that bound fields and their 4-potentials are auxiliary mathematical quantities, because one can write a Lagrangian of the system that is independent of these quantities.

An important attribute of the Breit interaction is that this expression takes a 
symmetric form with respect to a pair of interacting electrons. This kind of expression enables a consistent calculation of states of atomic electrons, where the electronic function takes an antisymmetric form which abides by the Pauli exclusion principle. The symbolic form of a general 2-electron interaction is

$$
H_{I n t}=\left\langle\Psi^{\dagger}\left|\sum_{i>j} \hat{O}_{i j}\right| \Psi\right\rangle
$$

Here $\Psi$ denotes an antisymmetric state of several electrons and $\hat{O}_{i j}$ is a symmetric operator which operates on the ijpair of electrons (see [19], p. 172).

The derivation of the electromagnetic energy-momentum tensor from the fields' Lagrangian density demonstrates another aspect of this issue. Here an application of the tensor $F^{\mu v}$ of (1), which is a sum of all kinds of electromagnetic fields, yields an unacceptable non-symmetric expression (see [2], pp. 86-87, [7], pp. 601-605). It is also shown in these textbooks how this erroneous result can be corrected by a mathematical trick. The unsatisfactory aspect of this approach has already been recognized [20] [21]. For example, the correction trick is based on a principle which states that it is legitimate to apply a procedure that arbitrarily alters local energy-momentum density. Obviously, such a procedure is inconsistent with general relativity.

It is proved in this work that radiation fields and bound fields represent different physical entities. Therefore, the calculation of the energy-momentum tensor from the sum of radiation fields and bound field is wrong. Moreover, it is shown above that bound fields do not represent an objective physical entity. Therefore, in order to derive the right expression for the energy-momentum tensor, one should use radiation fields only. It turns out that this calculation yields directly the correct expression [21]. This outcome demonstrates that if the starting point is correct then the result is correct and no further manipulations are required. The specific case discussed herein is that the separation of electromagnetic fields to radiation fields and bound fields is a fundamental attribute of the system, because it relies on experimental evidence. The correct form of the energy-momentum tensor which is described above provides another illustration of the generally valid opinion stating that consistent mathematics can correctly describe physical properties [1].

The claim that QED should be reconstructed may be regarded at odds with the standard approach that thinks that QED as an excellent theory. The following example of this opinion is taken from a textbook: "On the evidence presented in this table, QED is the most stringently tested-and the most dramatically successful —of all physical theories" (see [5], p. 198).

It turns out that eminent theoretical physicists have already asserted very serious qualms concerning the validity of such a statement. They refer to a fundamental QED process called renormalization. Feynman stated that renormalization is "a dippy process" (R. P. Feynman in [22], p. 128). P. A. M. Dirac has described it as a procedure of an "illogical character" [23]. He continued and said: "I am inclined to suspect that the renormalization theory is something that will 
not survive in the future, and that the remarkable agreement between its results and experiment should be looked on as a fluke". This approach is also mentioned in a textbook that makes the following statement: "In the quantum theory, these divergences do not disappear; on the contrary, they appear to get worse, and despite the comparative success of renormalisation theory the feeling remains that there ought to be a more satisfactory way of doing things" (see [24], p. 390).

It is interesting to note that it is now recognized that experimental evidence denies the QED's accuracy reputation [25]. Here the amazing QED precision of seven or more decimal digits dramatically deteriorates to just one decimal digit. This QED experimental failure indicates that Dirac was right with his negative opinion concerning the present structure of QED.

The analysis presented in this work proves new theoretical QED inconsistencies. These new results support the above mentioned negative opinions of eminent physicists concerning the present QED structure.

\section{Problems Related to the 4-Potential}

The foregoing analysis proves that the electromagnetic 4-potential $A_{\mu}(t, \boldsymbol{x})$ is not a consistent 4 -vector. In particular, this 4-potential cannot represent a fundamental physical entity. This state of affairs casts doubt on the validity of electromagnetic theoretical structures that are based on a 4-potential. Each of the following subsections contains a discussion of a specific case of this kind.

\subsection{Gauge Transformations}

Evidently, if the 4-potential does not represent a genuine physical entity then its gauge transformation cannot have a more profound meaning. And indeed, it has already been proved that gauge transformations contain many physical inconsistencies [26] [27] [28]. The following lines briefly describe few examples of this issue.

The primary justification of gauge transformation relies on the Dirac Lagrangian density (6). By definition, a gauge transformation uses a gauge function $\Lambda(x)$ which is an arbitrary function of the four space-time coordinates $x \equiv(t, \boldsymbol{x}) \quad$ (see [3], p. 342, [29], [30]). This transformation is defined as follows (see [3], p. $345,[5]$, p. 78, )

$$
A_{\mu}(x) \rightarrow A_{\mu}(x)+\Lambda(x)_{, \mu}, \quad \psi(x) \rightarrow \exp (-i e \Lambda(x)) \psi(x),
$$

where the symbol $e$ in the exponent denotes the electronic charge. A straightforward substitution of (12) into (6) proves that the Dirac Lagrangian density is invariant under this gauge transformation. Indeed, $\bar{\psi} \psi$ is a real quantity which is independent of the phase. Furthermore, the change of the 4-potential $A_{\mu}$ is canceled out by the corresponding quantity obtained from the partial derivative $i \partial_{\mu}$ of (6).

The consistency of the gauge transformation (12) is analyzed below. This 
analysis relies on the following theorem:

Theorem A: If a Lagrangian function is invariant under a certain transformation then the entire theory which is derived from this Lagrangian function is invariant under that transformation, provided the Lagrangian function and the transformation are free of mathematical contradictions.

The following points show two fundamental errors of the gauge transformation (12).

1) The power series expansion of the exponential function of (12) is

$$
\exp (-i e \Lambda(x))=1-i e \Lambda(x)+\cdots
$$

A basic law of physics says that all terms of a physically valid expression must have the same dimension. Furthermore, in the case of a relativistic expression, these terms must also undergo the same Lorentz transformation. The first term on the right-hand side of (13) is the pure number 1 , which is a dimensionless Lorentz scalar. The same is true for the imaginary number $i$, and in the units used herein also the electric charge $e$ is a dimensionless Lorentz scalar. It follows that the gauge function $\Lambda(x)$ must be a dimensionless Lorentz scalar. This constraint is violated by the intrinsic arbitrariness of the gauge function used by gauge theories (see [3], p. 342, [29], [30]). Moreover, it can be proved that a gauge function that is a dimensionless Lorentz scalar must be a numerical constant [26].

2) The de Broglie principle defines the relation between the wave length of a quantum particle and its momentum (see [31], p. 52)

$$
\boldsymbol{k}=\boldsymbol{p} / \hbar \text {. }
$$

It follows that an application of the quantum momentum operator $\boldsymbol{p}=-i \nabla$ to the exponential factor of (12) proves that in order to abide by the momentum value of the particle, the gauge function must be independent of the spatial coordinates. This result is inconsistent with the intrinsic arbitrariness of the gauge function used by gauge theories (see [3], p. 342, [29], [30]). In particular, [27] proves that contrary to experimental data, a gauge transformation destroys an interference pattern of an electronic beam.

The foregoing arguments hold for the domain of quantum mechanics. The following quotation proves that it also holds for quantum field theory (QFT). "First, some good news: quantum field theory is based on the same quantum mechanics that was invented by Schroedinger, Heisenberg, Pauli, Born, and others in 1925-26, and has been used ever since in atomic, molecular, nuclear and condensed matter physics" (see [3], p. 49).

This discussion proves that erroneous elements of gauge transformation exist in VE in general and in quantum theories in particular. Theorem A means that due to these errors, the invariance of the Lagrangian density (6) under a gauge transformation has no physical merit. As pointed out above, more details of problematic aspects of the gauge idea have already been published [26] [27] [28].

It is explained above why gauge transformations have no profound physical 
meaning. It is also pointed out in the Introduction that the 4-potential is not a part of MLE. Hence, in MLE the 4-potential and its gauge transformations are auxiliary quantities which can be used in mathematical procedures that aim to solve Maxwell equations. The following article presents a historical review of the development of gauge transformation ideas [32].

\subsection{The Dirac Monopole Theory}

The fundamental difference between an electric charge $e$ and a magnetic monopole (called briefly monopole) $g$ stems from the fact that an electric charge has been found in experiment whereas a corresponding monopole has not been found yet. Therefore, the monopole issue is a subject of theoretical work. Evidently, the first assignment is to define a theoretical expression for monopole. The following duality transformation of electromagnetic fields

$$
E \rightarrow B, \quad B \rightarrow-E,
$$

together with this charge-monopole transformation

$$
e \rightarrow g, \quad g \rightarrow-e
$$

are used for a monopole definition (see [7], pp. 251-252, [33], p. 1363). The transformations of the electromagnetic fields (15) can be put in a tensorial form

$$
F^{\mu \nu} \rightarrow F^{* \mu \nu}, F^{* \mu \nu} \rightarrow-F^{\mu \nu},
$$

where $F^{* \mu \nu}=\epsilon^{\mu \nu \alpha \beta} F_{\alpha \beta}$ and $\epsilon^{\mu \nu \alpha \beta}$ is the completely antisymmetric unit tensor of the fourth rank. The transformations (15) and (16) are sometimes called duality rotations by $\pi / 2$ (see [7], p. 252).

An application of the duality transformations (15) and (16) to the ordinary Maxwellian electrodynamics of a system that comprises electric charges and electromagnetic fields yields a dual theory of a system that comprises monopoles and electromagnetic fields. It means that the resultant theory holds for systems that contain no electric charge. Evidently, like the ordinary Maxwellian electrodynamics, the dual theory is regular at space-time points that are free of pointlike particles. Clearly, this outcome explains the monopole definition issue.

The next problem is to construct a unified charge-monopole theory of a system that comprises electric charges, monopoles and electromagnetic fields. This theory must satisfy the following requirements:

1) For systems of charges without monopoles, it must agree with the ordinary Maxwellian electrodynamics.

2) For systems of monopoles without charges it must agree with the dual theory which is described above.

A monopole theory has been constructed by Dirac [34] [35]. His theory relies on the idea that one and the same 4-potential is used for electromagnetic fields associated with charges and for electromagnetic fields associated with monopoles. A fundamental property of his theory are irregularities on strings of space-time points that are connected to every monopole. This outcome is inconsistent with the above mentioned regularity of the dual system of monopoles 
without charges. Hence, the Dirac theory violates requirement 2.

This theoretical contradiction is accompanied by a persistent failure of a very long list of experimental searches for a Dirac monopole. Indeed, the official PDG site recently published a review of monopole search and concludes: "To date there have been no confirmed observations of exotic particles possessing magnetic charge" [36]. This systematic failure is yet another example of the inherent relations between consistent mathematics and experimental evidence [1].

The following issue pertains to the discrepancies of the Dirac monopole theory. Wikipedia is a well-known source of information. According to its principles, it describes ideas that are consistent with the current consensus. Thus, the electric charge Wikipedia item describes properties of one kind of physical objects (which can be either positive or negative). This evidence together with the above mentioned duality transformations (15), (16) prove that one kind of monopole should exist. By contrast, the Wikipedia monopole item describes several and different kinds of monopoles. This state of affairs shows that the opinion of the present community is yet unsettled with respect to the Dirac monopole concept.

It is interesting to point out that a very long time ago scientific articles have already predicted the failure of the quest for a Dirac monopole [37] [38]. The foregoing PDG monopole report proves that as of today, these predictions are correct. The mathematical problems of the Dirac monopole theory support the expectation that no genuine Dirac monopole will be found in experiment.

\subsection{The Aharonov-Bohm Effects}

The original $A B$ article [39] presents two quantum mechanical effects called the electric $\mathrm{AB}$ effect and the magnetic $\mathrm{AB}$ effect. These authors discuss a system of an electron (called herein the traveling electron) that interacts with a macroscopic source of a 4-potential. The beam of the traveling electron splits into two sub-beams where each of which moves in a field-free region. Later, the sub-beams produce an interference pattern. Relying on their analysis, $A B$ argue that the 4-potential is an indispensable element of quantum mechanics. Indeed, in the summary section of [39] they state: "The essential result of the previous discussion is that in quantum theory, an electron (for example) can be influenced by the potentials even if all the field regions are excluded from it. In other words, in a field-free multiply-connected region of space, the physical properties of the system still depend on the potentials." Hereafter, this statement is called the main $A B$ assertion.

The following argument proves that the main $\mathrm{AB}$ assertion cannot be correct. Indeed, $A B$ discuss systems that do not contain radiation fields. Therefore, the Breit interaction presented in section 6 can be used as a basis for a quantum mechanical analysis of the problem. In this case the interaction of the traveling electron with the source can be cast into the form of a sum of two body-interaction of the traveling electron with each charge of the source. This application of the Breit interaction means that the problem can be legitimately 
reduced to a mechanical-like Lagrangian where the potentials and the fields are not directly used. Therefore, the $\mathrm{AB}$ potentials are nothing more than auxiliary quantities that play no fundamental role. This argument refutes the main $\mathrm{AB}$ assertion. Few details of this general conclusion are presented below.

- The first $A B$ article [39] treats a single particle problem of the traveling electron that moves in an external potential. This is inconsistent with the Breit interaction which proves that the interaction takes the symmetric sum of a two-body interaction. It means that [39] ignores the contribution of the source. Later $\mathrm{AB}$ admitted this problem but argue that an inclusion of the source does not change the results [40]. The following arguments prove that this $\mathrm{AB}$ conclusion is unjustified.

- It has been proved that a crucial element of the existence of the $A B$ effects is that the source should behave as an inert object [41] [42]. Namely, the same 4-potentials yield different interference patterns if the source is inert or non-inert. This outcome demonstrates the crucial role of the source and refutes the main $\mathrm{AB}$ assertion.

- The symmetric form of the Breit two-charge interaction means that the interaction may be cast either to the interaction of the traveling electron with the potential of the source or to the interaction of the source with the 4-potential of the traveling electron. These two forms are equivalent. In the latter case, the fields of the traveling electron do not vanish at the source. It means that in this legitimate description there is no field-free region and a fortiori no multiply-connected field-free region exists. Hence, the main $\mathrm{AB}$ assertion is refuted. It is interesting to note that several authors have recently claimed that the inclusion of the source denies the main $\mathrm{AB}$ assertion concerning field-free potentials [43] [44] [45]. These claims are in accordance with the analysis presented earlier in [41] [42].

- It has also been proved that the electric $A B$ effect is based on arguments that lead to a violation of the law of energy conservation [46] [47]. This unacceptable contradiction substantiates the claim that the main $A B$ assertion is inherently wrong.

It is interesting to note that the experimental confirmation of the magnetic $\mathrm{AB}$ effect uses an inert source of the vector potential $\boldsymbol{A}$ [48]. The result of this experiment agrees with the analysis of [41] [42].

\section{Conclusions}

Section 2 presents contradictory quotations from respectable textbooks that demonstrate the extremely problematic status of the electromagnetic 4-potential $A_{\mu}(t, \boldsymbol{x})$. The present work analyzes the theoretical meaning of this quantity. Obviously, in MLE, the 4-potential is an auxiliary quantity, because the primary equations of this theory-namely, Maxwell equations and the Lorentz law of force-are independent of the 4-potential. As is well known, $A_{\mu}(t, \boldsymbol{x})$ is useful in attempts to solve problems (see [7], p. 219). It means that the usefulness of the 4-potential $A_{\mu}(t, \boldsymbol{x})$ cannot be denied. 
This work is dedicated to an analysis of the theoretical significance of the 4-potential $A_{\mu}(t, \boldsymbol{x})$ in VE. This paper proves that like the case of MLE, in VE the 4-potential is an auxiliary quantity. An important element of the discussion is the proof that radiation fields and bound fields are inherently different physical objects. This evidence means that the present QED form should be revised. It is also proved above that the radiation 4-potential is not a 4-vector of the form $A_{\mu}(t, \boldsymbol{x})$. An analogous argument can already be found in a well-known textbook (see [3], p. 251). It is explained why the dependence of the Lienard-Wiechert 4-potential on retarded coordinates is inconsistent with the required locality of functions used by a Lagrangian density. It is also shown that relativistic covariance of electrodynamics can be consistently restored if in each frame radiation fields are used for a reconstruction of a 4-potential. This procedure directly yields a 4-potential whose form is required for QED analysis (9).

Bound fields have different properties. Here the Darwin Lagrangian and the associated Breit interaction prove that the Lagrangian takes a mechanical-like form that depends on instantaneous values of the coordinates and the velocities, but is independent of the fields and of their 4-potential. Hence, in the case of bound fields, both the electromagnetic fields and their 4-potentials are auxiliary quantities. The time-independence of the Breit interaction is consistent with the standard practice of atomic calculations which use functions that take the form of the time-independent Heisenberg picture.

It is also proved that some physical concepts that are based on the 4-potentials are unjustified. These issues include the idea of gauge transformations in VE, the Dirac monopole theory and the two AB effects.

\section{Conflicts of Interest}

The author declares no conflicts of interest regarding the publication of this paper.

\section{References}

[1] Wigner, E. (1960) The Unreasonable Effectiveness of Mathematics in the Natural Sciences. Richard Courant Lecture in Mathematical Sciences Delivered at New York University, May 11, 1959. Communications on Pure and Applied Mathematics, 13, 1-14. https://doi.org/10.1002/cpa.3160130102

[2] Landau, L.D. and Lifshitz, E.M. (2005) The Classical Theory of Fields. Elsevier, Amsterdam.

[3] Weinberg, S. (1995) The Quantum Theory of Fields, Vol. I. Cambridge University Press, Cambridge. https://doi.org/10.1017/CBO9781139644167

[4] Bjorken, J.D. and Drell, S.D. (1965) Relativistic Quantum Fields. McGraw-Hill, New York.

[5] Peskin, M.E. and Schroeder, D.V. (1995) An Introduction to Quantum Field Theory. Addison-Wesley, Reading, Mass.

[6] Griffiths, D. (2008) Introduction to Elementary Particles. 2nd Edition, Wiley-VCH, Weinheim. 
[7] Jackson, J.D. (1975) Classical Electrodynamics. 2nd Edition, John Wiley, New York.

[8] Immanuel Kant (2018). https://en.wikipedia.org/wiki/Immanuel_Kant

[9] Wu, A.C.T. and Yang, C.N. (2006) Evolution of the Concept of the Vector Potential in the Description of Fundamental Interactions. International Journal of Modern Physics A, 21, 3235-3277. https://doi.org/10.1142/S0217751X06033143

[10] Feynman, R.P., Leighton, R.B. and Sands, M. (1965) The Feynman Lectures on Physics, V. II. Addison-Wesley, Reading, Mass.

[11] Schiff, L.I. (1955) Quantum Mechanics. McGraw-Hill, New York.

[12] Patrignani, C., et al. (Particle Data Group) (2016) Review of Particle Physics. Chinese Physics C, 40, Article ID: 1100001.

[13] Comay, E. (2018) Inherent Differences between Bound and Radiation Fields. OALib, 5, e4517. https://www.scirp.org/journal/PaperInformation.aspx?PaperID=83992

[14] Wigner, E. (1939) On Unitary Representations of the Inhomogeneous Lorentz Group. Annals of Mathematics, 40, 149-204. https://doi.org/10.2307/1968551

[15] Schweber, S.S. (1964) An Introduction to Relativistic Quantum Field Theory. Harper \& Row, New York, 44-53.

[16] Comay, E. (2018) Lorentz Transformation of Radiation 4-Potential. Acta Physica Polonica A, 133, 1294-1298. https://doi.org/10.12693/APhysPolA.133.1294

[17] Bethe, H.A. and Salpeter, E.E. (1957) Quantum Mechanics of One- and Two-Electron Atoms. Springer, Berlin.

[18] Bjorken, J.D. and Drell, S.D. (1964) Relativistic Quantum Mechanics. McGraw-Hill, New York.

[19] Condon, E.U. and Shortley, G.H. (1964) The Theory of Atomic Spectra. University Press, Cambridge.

[20] Munoz, G. (1996) Lagrangian Field Theories and Energy-Momentum Tensors. American Journal of Physics, 64, 1153. https://doi.org/10.1119/1.18336

[21] Comay, E. (2018) A Consistent Construction of the Electromagnetic Energy-Momentum Tensor. Open Access Library Journal, 5, 1-8. http://www.scirp.org/journal/PaperInformation.aspx?paperID=82391

[22] Feynman, R.P. (1990) QED, the Strange Theory of Light and Matter. Penguin, London.

[23] Dirac, P.A.M. (1963) The Evolution of the Physicist's Picture of Nature. Scientific American, 208, 45-53. https://doi.org/10.1038/scientificamerican0563-45

[24] Ryder, L.H. (1997) Quantum Field Theory. Cambridge University Press, Cambridge.

[25] Pohl, R., et al. (2010) The Size of the Proton. Nature, 466, 213-216. https://doi.org/10.1038/nature09250

[26] Comay, E. (2008) Mathematical Constraints on Gauge in Maxwellian Electrodynamics. Apeiron, 15, 123. https://www.tau.ac.il/ elicomay/GAUGE.pdf

[27] Comay, E. (2016) A New Quantum Paradox. Physical Science International Journal, 12, 1. http://www.sciencedomain.org/abstract/16442 https://doi.org/10.9734/PSIJ/2016/28572

[28] Comay, E. (2017) Gauge Contradictions in the QED Lagrangian Density. Open Access Library Journal, 4, 1-7. http://www.scirp.org/journal/PaperInformation.aspx?PaperID=76182

[29] Pauli, W. (1941) Relativistic Field Theories of Elementary Particles. Reviews of 
Modern Physics, 13, 203-232. https://doi.org/10.1103/RevModPhys.13.203

[30] Yang, C.N. and Mills, R. (1954) Conservation of Isotopic Spin and Isotopic Gauge Invariance. Physical Review, 96, 191-196. https://doi.org/10.1103/PhysRev.96.191

[31] Messiah, A. (1967) Quantum Mechanics. Vol. 1, North Holland, Amsterdam.

[32] Jackson, J.D. and Okun, L.B. (2001) Historical Roots of Gauge Invariance. Reviews of Modern Physics, 73, 663-680.

[33] Goddard, P. and Olive, D.I. (1978) Magnetic Monopoles in Gauge Field Theories. Reports on Progress in Physics, 41, 1357-1437. https://doi.org/10.1088/0034-4885/41/9/001

[34] Dirac, P.A.M. (1931) Quantised Singularities in the Electromagnetic Field. Proceedings of the Royal Society A, 133, 60-72. https://doi.org/10.1098/rspa.1931.0130

[35] Dirac, P.A.M. (1948) The Theory of Magnetic Poles. Physical Review, 74, 817-830. https://doi.org/10.1103/PhysRev.74.817

[36] http://pdg.lbl.gov/2018/reviews/rpp2018-rev-mag-monopole-searches.pdf

[37] Zwanziger, D. (1965) Dirac Magnetic Poles Forbidden in S-Matrix Theory. Physical Review, 137, 647-648. https://doi.org/10.1103/PhysRev.137.B647

[38] Comay, E. (1985) Will Magnetic Monopoles Be Detected in Our Instruments. Lettere al Nuovo Cimento, 43, 150-152. https://doi.org/10.1007/BF02749596

[39] Aharonov, Y. and Bohm, D. (1959) Significance of Electromagnetic Potentials in the Quantum Theory. Physical Review, 115, 485-491. https://doi.org/10.1103/PhysRev.115.485

[40] Aharonov, Y. and Bohm, D. (1961) Further Considerations on Electromagnetic Potentials in the Quantum Theory. Physical Review, 123, 1511-1524. https://doi.org/10.1103/PhysRev.123.1511

[41] Comay, E. (2000) Interrelations between the Neutron's Magnetic Interactions and the Magnetic Aharonov-Bohm Effect. Physical Review A, 62, Article ID: 042102. https://doi.org/10.1103/PhysRevA.62.042102

[42] https://arxiv.org/pdf/0910.3289.pdf

[43] Vaidman, L. (2012) Role of Potentials in the Aharonov-Bohm Effect. Physical Review $A$, 86, Article ID: 040101. https://doi.org/10.1103/PhysRevA.86.040101

[44] Pearle, P. and Rizzi, A. (2017) Quantum-Mechanical Inclusion of the Source in the Aharonov-Bohm Effects. Physical Review A, 95, Article ID: 052123. https://doi.org/10.1103/PhysRevA.95.052123

[45] Pearle, P. and Rizzi, A. (2017) Quantized Vector Potential and Alternative Views of the Magnetic Aharonov-Bohm Phase Shift. Physical Review A, 95, Article ID: 052124. https://doi.org/10.1103/PhysRevA.95.052124

[46] Comay, E. (1987) Conservation Laws and the Electric Aharonov-Bohm Effect. Physics Letters A, 120, 196-198. https://doi.org/10.1016/0375-9601(87)90335-5

[47] Comay, E. (1987) Further Comments on the Original Derivation of the Electric Aharonov-Bohm Effect. Physics Letters A, 125, 403-404. https://doi.org/10.1016/0375-9601(87)90170-8

[48] Tonomura, A, et al. (1986) Evidence for Aharonov-Bohm Effect with Magnetic Field Completely Shielded from Electron Wave. Physical Review Letters, 56, 792-795. https://doi.org/10.1103/PhysRevLett.56.792 